\title{
Colorantes alimentarios y su relación con la salud: ¿cómo abordar esta problemática desde el estudio de las disoluciones?
}

\author{
Rocío Belén Kraser \\ Gabinete de Didáctica de la Química, Departamento de Química, Universidad Nacional del Sur. Babia \\ Blanca,Buenos Aires, Argentina.rbkraser@gmail.com \\ ORCID: https:/ / orcid.org/0000-0001-9677-6361
}

Sandra Analía Hernández

Gabinete de Didáctica de la Química, Departamento de Química, Universidad Nacional del Sur. INQUISUR (UNS-CONICET). Babia Blanca, Buenos Aires, Argentina.

sandra.bernandez@uns.edu.ar

ORCID: https://orcid.org/0000-0002-1188-1101

[Recibido: 30 Abril 2019. Revisado: 10 Septiembre 2019. Aceptado: 13 Octubre 2019]

\begin{abstract}
Resumen: Estudios realizados han demostrado que los/las adolescentes, en su mayoría, consumen de manera frecuente cantidades considerables de bebidas a base de jugos en polvo, los cuales contienen colorantes alimentarios potencialmente nocivos para la salud. En tal sentido, se aborda esta problemática desde el estudio de las disoluciones. La propuesta didáctica en contexto se llevó a cabo con estudiantes de segundo año de educación secundaria con el objetivo no solo de trabajar la temática curricular, sino también con la intención de contribuir a la educación para la salud y a la concientización de los jóvenes en su rol de consumidores. A través del trabajo contextualizado y de las actividades teórico-prácticas propuestas, se logró el abordaje del tema disoluciones poniendo en consideración el estudio de los colorantes alimentarios presentes en jugos en polvo y su relación con la salud. La estrategia metodológica aplicada resultó útil para sortear las dificultades que los/las estudiantes suelen manifestar al abordar el tema disoluciones y para enfatizar la importancia del trabajo experimental y la contextualización.
\end{abstract}

Palabras clave: Disoluciones químicas; Colorantes alimentarios; Jugos en polvo; Salud; Aprendizaje en contexto.

Artificial dyes and their relation to health: how to approach this problem from the study of solutions?

Abstract: Studies have shown that adolescents, for the most part, frequently consume considerable amounts of drinks based on powdered juices which contain food dyes potentially harmful to health. In this sense, this problem is addressed from the study of dissolutions. The didactic proposal in context was carried out with second year students of secondary education with the objective not only of working on the curricular theme, but also with the intention of contributing to education for health and to the awareness of young people in their role of consumers. Through the contextualized work and the proposed theoretical-practical activities, the approach of the dissolutions theme was achieved by considering the study of the food dyes present in powdered juices and their relationship with health. The applied methodological strategy was useful to overcome the difficulties that students usually manifest when dealing with the topic of dissolutions and to emphasize the importance of experimental work and contextualization.

Keywords: Chemical solutions; Food dyes; Powdered juice; Health; Learning in context.

Para citar este artículo: Kraser R. B., Hernández S. A. (2020) Colorantes alimentarios y su relación con la salud: ¿cómo abordar esta problemática desde el estudio de las disoluciones? Revista Eureka sobre Enseñanza y Divulgación de las Ciencias 17 (1), 1202. doi: 10.25267/Rev_Eureka_ensen_divulg_cienc.2020.v17.11.1202 


\section{Introducción}

La contextualización en las clases de ciencias constituye un aspecto primordial para la comprensión de los conceptos científicos y la interpretación de su relación con la vida cotidiana (Broman y Parchmann 2014, Marchán-Carvajal y Sanmartí 2014, Caamaño 2018).

Cuando se habla de contexto se refiere al escenario que se escoge para generar en el alumnado la necesidad de entender alguno de los fenómenos que pasan o han pasado en el mundo. Su comprensión, a través de la construcción y uso de unas ideas científicas y sobre la ciencia, debería capacitar a los aprendices para actuar en el marco de una comunidad de práctica (Sanmartí y Márquez 2017, pp. 6-7).

En este sentido, el estudio de las disoluciones resulta de gran interés en la enseñanza de la química, no sólo porque las mismas son ampliamente utilizadas en el laboratorio químico, sino también porque forman parte de nuestra vida diaria. Encontramos disoluciones cotidianamente en formulaciones alimenticias, farmacéuticas, de higiene y limpieza e incluso en nuestro propio organismo.

No obstante su importancia, diversas investigaciones didácticas han puesto de manifiesto las dificultades detectadas en la comprensión de los conceptos asociados al tema disoluciones por parte de estudiantes tanto de educación secundaria (Blanco López y Prieto Ruz 1994) como de los primeros años de universidad (Ricchi y Torres 2014).

Al respecto, Nappa, Insausti y Sigüenza (2005) señalan que "la realidad del aula muestra que existen obstáculos conceptuales que impiden una generación adecuada de representaciones mentales sobre disoluciones $y$, consecuentemente, el aprendizaje no se concreta eficientemente".

El estudio de los colorantes alimentarios presentes en disoluciones de jugo en polvo en agua, constituye una buena oportunidad para el aprendizaje integral de los/las estudiantes, ya que no sólo favorece al entendimiento de fenómenos químicos y físicos en contexto, sino que también brinda la posibilidad de incluir la educación para el consumidor como contenido transversal en las clases de ciencia (Ruiz Hidalgo 2009).

En nuestro entorno de trabajo, los resultados obtenidos a partir de un relevamiento estadístico pusieron de manifiesto que la mala calidad de agua y su escasez ocasional, han llevado a la población de nuestra ciudad hacia el consumo de distintos tipos de bebidas tales como gaseosas, aguas saborizadas, jugos y aguas envasadas (Brizuela y Hernández 2016). En particular, una encuesta realizada a 178 jóvenes de entre 13 y 19 años puso en evidencia que la mayoría de los adolescentes consultados consume jugos en polvo, bebidas gaseosas y aguas saborizadas en su dieta diaria; siendo los jugos el polvo diluidos en agua las bebidas más escogidas por los estudiantes $(66,8 \%)$. Por otra parte, la mayoría de los alimentos consumidos por niños y jóvenes (snacks, gaseosas, jugos, golosinas, etcétera) contienen una amplia variedad de colorantes artificiales (Sánchez 2013), algunos de los cuales han sido estudiados científicamente demostrando ocasionar problemas en la salud de los consumidores, principalmente si se tiene en cuenta la frecuencia y el exceso en su consumo (Burrows 2009, Cheeseman 2012 Cúneo y Schaab 2013, León Espinosa 2000).

\section{Objetivos}

Son objetivos de esta propuesta:

- estudiar el tema disoluciones a través de la contextualización y la problematización;

- estudiar los colorantes alimentarios presentes en disoluciones de jugos en polvo y su incidencia en la salud; 
- abordar la educación para la salud y para el consumidor como un contenido transversal en las clases de ciencia brindando a los jóvenes herramientas que les permitan tomar decisiones responsables;

- alentar a los docentes de ciencias a emplear metodologías de trabajo contextualizadas que promuevan un aprendizaje significativo de los contenidos a abordar;

- contribuir a la formación de ciudadanos críticos y reflexivos.

\section{Acerca de los colorantes alimentarios}

A la hora de escoger un alimento, si bien son varios los aspectos que un consumidor evalúa (sabor, precio, información nutricional), el color suele ser determinante e influye en gran medida en la aceptación o rechazo de dicho alimento. Los colorantes tanto naturales como artificiales forman parte del amplio grupo de los aditivos alimentarios. El Codex Alimentarius (1995) define a un aditivo alimentario de la siguiente manera:

"Cualquier sustancia que en cuanto tal no se consume normalmente como alimento, ni tampoco se usa como ingrediente básico en alimentos, tenga o no valor nutritivo, y cuya adición intencionada al alimento con fines tecnológicos (incluidos los organolépticos) en sus fases de fabricación, elaboración, preparación, tratamiento, envasado, empaquetado, transporte o almacenamiento, resulte o pueda preverse razonablemente que resulte (directa o indirectamente) por sí o sus subproductos, en un componente del alimento o un elemento que afecte a sus características. Esta definición no incluye "contaminantes" o sustancias añadidas al alimento para mantener o mejorar las cualidades nutricionales" (Codex Alimentarius 1995, p. 3).

Los colorantes alimentarios no sólo cumplen la función de conferir color a un alimento, sino también de intensificar o restaurar el color perdido ya sea por las condiciones dadas durante el procesado o el almacenamiento (luz, aire, temperatura, presencia de otros componentes del alimento, etcétera). Buera (2011) explicita que "el agregado de colorantes ayuda a que ciertos alimentos tengan aspecto agradable, mejorando su aspecto visual y dando así respuesta a las expectativas del consumidor".

Este tipo de aditivos, ya sea en su forma natural o artificial, representan un interesante conjunto de sustancias de consumo diario de gran incidencia en nuestras vidas, las cuales merecen especial atención dado que algunos han sido relacionados con posibles efectos adversos en la salud (Burrows 2009, Cheeseman 2012, León Espinosa 2000).

\section{Marco curricular}

La propuesta presentada en este trabajo plantea el abordaje del tema disoluciones en el nivel secundario de escolarización. Se propone contextualizar su estudio a los preparados de jugos en polvo, abordando de manera transversal a los colorantes alimentarios presentes en estas bebidas y su relación con la salud. Se trabajó con estudiantes de segundo año en la materia Fisicoquímica, dentro del eje temático: la naturaleza corpuscular de la materia el cual a su vez comprende el estudio de: los estados de la materia, las disoluciones y los cambios físicos y químicos. Dentro de dicha unidad temática, se abordan además los métodos de fraccionamiento de los componentes de una disolución.

\section{Diagnóstico previo. Verificando la contextualización}

Con el objetivo de conocer los hábitos de consumo y el nivel de concientización de los/las estudiantes respecto al consumo y composición de los jugos en polvo y en línea con la metodología de trabajo a desarrollar, se realizó un cuestionario ad hoc el cual fue respondido 
por veintidós estudiantes pertenecientes a segundo año de educación secundaria que acreditaron tener entre 12 y 14 años de edad.

El cuestionario fue diseñado por la docente del curso en un formulario de Google Drive, cuyo link fue distribuido a través de WhatsApp al resto de los/las estudiantes quienes lo completaron, durante la clase, desde sus celulares.

El mismo estuvo destinado a sondear el consumo de bebidas a base de jugos en polvo, la frecuencia de consumo y el hábito de lectura de las etiquetas de los alimentos consumidos.

La encuesta realizada evidenció que un 77,3\% de los/las estudiantes consume bebidas a base de jugos en polvo; mientras que el 22,7 \% dijo no consumir este tipo de bebidas.

En relación a la frecuencia en el consumo de bebidas a base de jugos en polvo, un 58,8 \% de los jóvenes manifestó consumir de manera frecuente, mientras que un $41,2 \%$ consumen esporádicamente.

En lo que respecta a la lectura del etiquetado, la mayor parte de los/las estudiantes $(59,1 \%)$ expresó no leer "nunca" la información brindada por los rótulos de los alimentos consumidos mientras que un 40,9 \% declaró que sólo "algunas veces" lo hacen. Quienes declararon leer el etiquetado dijeron que ponían especial énfasis en la lectura de las fechas de elaboración y/o vencimiento del alimento y de su información nutricional.

Este primer sondeo permitió verificar la posible contextualización del tema disoluciones a partir de preparados de jugos en polvo. La consulta acerca de la marca de jugo preferentemente consumido con mayor frecuencia por los/las estudiantes permitió consensuar acerca del jugo en polvo a utilizar durante el desarrollo de la propuesta a trabajar.

\section{Descripción de la propuesta}

Para llevar a cabo esta propuesta se recurre a diversos recursos y estrategias metodológicas las cuales se irán presentando y explicando sus alcances de acuerdo a la siguiente secuencia de actividades: a) Ideas previas y preconcepciones; b) Lectura y análisis de etiquetas de jugos en polvo.; c) Trabajo práctico experimental; d) Estudio de los colorantes presentes en jugo de naranja en polvo; e) Sociabilización de lo trabajado.

\section{Ideas previas y preconcepciones}

Las ideas o saberes previos, son aquellos conocimientos que el aprendiz ya sabe acerca de los contenidos que se desean abordar y aunque algunas veces suelen ser parciales o erróneos, son los que el/la estudiante utiliza para vincular un nuevo conocimiento a aprender (Ausubel 1963, Díaz-Barriga y Hernández Rojas 2002, Limon y Carretero 1997). En sus reflexiones acerca de por qué la ciencia es tan difícil de aprender, Johnstone (1991) postula que: "Lo que realmente sabemos y entendemos controla lo que aprendemos."

En tal sentido, con el objetivo de conocer las ideas previas de los/las estudiantes respecto al tema a estudiar, se trabajó con diversas imágenes que permitieron abordar conceptos tales como: sustancias puras, mezclas homogéneas, mezclas heterogéneas, fases y componentes. Para recuperar el concepto de mezclas desde su influencia en la vida diaria, se utilizaron como recursos disparadores imágenes de productos de uso cotidiano (productos alimenticios, de higiene, de limpieza, entre otros). A partir de las mismas se les pidió a los/las alumnos/as que realizaran una posible clasificación de las imágenes dadas en función de su composición y aspecto. Esto permitió ahondar en los conceptos de fases y componentes y elaborar una definición de mezclas homogéneas y heterogéneas. Posteriormente, se les pidió a los/las estudiantes que propongan otros ejemplos de mezclas de uso cotidiano. En esta instancia, los/las alumnos/as participaron activamente y propusieron algunos de los siguientes ejemplos: 
"la leche chocolatada", "la masa de una torta", "una ensalada", "una gaseosa", los cuales fueron descriptos y clasificados.

A continuación, se les presentó a los/las estudiantes una nueva imagen en la que se puede observar un sobre de jugo en polvo y se debatió acerca de si el contenido del sobre constituía o no una mezcla. Como resultado del debate, $1 / 3$ del curso expresó que no se trataba de una mezcla ya que era "sólo jugo", por lo tanto constituía una sustancia pura; mientras que el resto del grupo clase coincidió en que el contenido del sobre constituía una mezcla por contener más de un componente.

\section{Lectura y análisis de etiquetas de jugos en polvo}

Como quedó evidenciado en el diagnóstico previo, existe cierto desconocimiento por parte de los consumidores encuestados respecto a la información que brinda un rótulo en un alimento. La etiqueta o rótulo, se encuentra adherida al envase de un alimento y tiene la función de brindar al consumidor información sobre las características de un alimento. Obligatoriamente debería figurar en un rótulo: denominación de venta del alimento; lista de ingredientes; contenidos netos; identificación del origen; nombre o razón social, y dirección del exportador cuando corresponda; identificación del lote; fecha de duración; preparación e instrucciones de uso del alimento, cuando corresponda; rotulado nutricional (OMS/FAO 2007, Codex Alimentarius 2007).

A partir de la propuesta descripta a continuación, se propone trabajar en la lectura crítica y análisis de etiquetas de jugos en polvo de diversas marcas y sabores, con el objetivo de educar en la salud y como consumidores a los adolescentes y fomentando una actitud responsable. Se pretende que los/las estudiantes sean capaces de reconocer y analizar la información que brindan los rótulos como así también de identificar las diversas sustancias presentes: edulcorantes, agentes emulsificantes, antioxidantes, acidulantes, reguladores de acidez, edulcorantes no nutritivos, antiaglutinantes, aromatizantes, espesantes y colorantes.

A través del estudio de la composición de los jugos y poniendo énfasis en los colorantes presentes, pudieron detectar que existen reglamentaciones específicas en relación con el rotulado de ciertos aditivos, como el colorante artificial tartrazina, el cual además de estar listado como colorante, tiene una leyenda aclaratoria escrita con caracteres de buen tamaño, realce y visibilidad que dice: "CONTIENE TARTRAZINA", lo cual permite abrir el debate acerca de los colorantes y sus efectos en la salud de los consumidores.

A través de esta propuesta los/las estudiantes pudieron reflexionar acerca de la gran cantidad de alimentos que consumimos a diario aditivados con colorantes y que no consta en la mayoría de las etiquetas la cantidad de colorante agregado.

\section{Trabajo práctico experimental}

Para el desarrollo de las tareas experimentales se utilizó como sustancia de estudio el jugo en polvo del sabor y de la marca más elegida por los encuestados.

El trabajo experimental se dividió en dos partes: en la primera parte se prepararon cuatro disoluciones de jugo de naranja en polvo y agua que permitieron trabajar con expresiones de concentración; en la segunda parte se realizó el análisis cualitativo de los colorantes presentes en el jugo en polvo utilizando la cromatografía como método de fraccionamiento de los componentes de una disolución. 


\section{Primera parte: Preparación de disoluciones en el laboratorio}

Los/las estudiantes trabajaron en grupos en la preparación de disoluciones de diversas concentraciones de jugo en polvo sabor naranja. Para ello, dispusieron cuatro vasos de precipitados, previamente enumerados, a cada uno de los cuales agregaron $30 \mathrm{~mL}$ de agua medidos con probeta. En el primero, segundo, tercero y cuarto vaso; agregaron una, tres, cinco y nueve cucharadas de jugo en polvo, respectivamente. Una vez preparadas las disoluciones, los estudiantes registraron sus observaciones e infirieron, en primera instancia, el grado de concentración de las mismas a partir de la percepción visual y olfativa. De esta manera, se observaron y compararon algunas de las propiedades intensivas de las diluciones obtenidas (color y olor), para poder establecer una primera aproximación hacia los conceptos de soluciones diluidas y concentradas.

Para poder realizar los cálculos de concentración correspondientes a cada una de las cuatro disoluciones preparadas, se pesó la masa de jugo en polvo equivalente a una cucharada colmada. Se empleó una cuchara-espátula metálica de laboratorio de tamaño pequeño. Por lo tanto, se estimó que la masa de una cucharada de jugo en polvo equivalía a $0,1 \mathrm{~g}$. A partir de dicha masa, el volumen de agua agregado y considerando el valor de la densidad del agua como $1 \mathrm{~g} / \mathrm{mL}$, se realizaron los cálculos de la unidad física de concentración porcentaje masa en masa $(\% \mathrm{~m} / \mathrm{m})$ como muestra.

El porcentaje de masa de soluto en masa de disolución $(\% \mathrm{~m} / \mathrm{m})$ representa la masa en gramos de soluto que están disueltos en $100 \mathrm{~g}$ de disolución.

Teniendo en cuenta la ecuación (1), que define la unidad física de concentración de porcentaje masa en masa, se pueden calcular de manera sencilla las concentraciones de las disoluciones preparadas.

$$
\% \mathrm{~m} / \mathrm{m}=[\text { masa de soluto } / \text { (masa de soluto }+ \text { masa de disolvente) }] \times 100 \text {. }
$$

Una vez calculadas todas las concentraciones se tabularon (tabla 1) y se realizó una puesta en común de los valores obtenidos. De esta manera, los/las estudiantes lograron establecer una correspondencia entre los registros iniciales y los valores de la unidad física de concentración calculada.

Tabla 1. Datos y concentración de cada disolución preparada expresada como porcentaje masa en masa.

\begin{tabular}{|c|c|c|c|}
\hline Disolución preparada & Masa de soluto & Masa de solvente & Masa de disolución \\
\hline Disolución $\mathrm{N}^{\circ} 1$ & $0,1 \mathrm{~g}$ & $30 \mathrm{~g}$ & $30,1 \mathrm{~g}$ \\
\hline Disolución $\mathrm{N}^{\circ} 2$ & $0,3 \mathrm{~g}$ & $30 \mathrm{~g}$ & $30,3 \mathrm{~g}$ \\
\hline Disolución $\mathrm{N}^{\circ} 3$ & $0,5 \mathrm{~g}$ & $30 \mathrm{~g}$ & $30,5 \mathrm{~g}$ \\
\hline Disolución $\mathrm{N}^{\circ} 4$ & $0,9 \mathrm{~g}$ & $30 \mathrm{~g}$ & $30,9 \mathrm{~g}$ \\
\hline
\end{tabular}

Como puede verse en la tabla 1 , hasta aquí los estudiantes trabajaron con un mismo volumen de solvente y distinta cantidad de soluto. Luego de discutir al respecto, se les propone el siguiente desafío:

¿Qué volumen de agua debería agregar a la disolución $N^{\circ} 4$ para que la concentración de la nueva dilución fuera igual a la de la disolución $N^{\circ} 1$ ?

En función de los datos de concentración de las disoluciones $\mathrm{N}^{\circ} 1$ y $\mathrm{N}^{\circ} 4$ preparadas mostrados en la tabla 1 y teniendo en cuenta la definición de la unidad física de concentración de porcentaje masa en masa, se puede resolver de manera sencilla este desafío.

De la ecuación 1 sabemos que:

$$
\% \mathrm{~m} / \mathrm{m}=[\text { masa de soluto } / \text { (masa de soluto }+ \text { masa de disolvente })] \times 100 .
$$


A su vez, sabemos que: el porcentaje de masa de soluto en masa de disolución $(\% \mathrm{~m} / \mathrm{m})$ representa la masa en gramos de soluto que están disueltos en $100 \mathrm{~g}$ de disolución.

Es decir que para la disolución $\mathrm{N}^{\circ} 1$ la concentración sea de $0,33 \% \mathrm{~m} / \mathrm{m}$, significa que:

$$
0,33 \% \mathrm{~m} / \mathrm{m}=0,33 \mathrm{~g} \text { soluto } / 100 \mathrm{~g} \text { disolución. }
$$

Teniendo en cuenta esta expresión y los datos de soluto y disolvente utilizados para preparar la disolución $\mathrm{N}^{\circ} 2$, se puede calcular la cantidad de agua que debería agregar a la disolución $\mathrm{N}^{\circ} 4$ para que la concentración de la nueva dilución fuera igual a la de la disolución $\mathrm{N}^{\circ} 1$.

Así:

$$
0,33 \mathrm{~g} \text { soluto } / 100 \mathrm{~g} \text { disolución }=0,9 \mathrm{~g} \text { soluto } /(0,9 \mathrm{~g} \text { soluto }+ \text { masa total de disolvente })
$$

Despejando se obtiene que la masa total de disolvente necesaria sería 271,83 g.

Dado que para preparar las disoluciones utilizamos $30 \mathrm{~g}$ de agua $\left(30 \mathrm{~mL} ; \rho_{\text {agua }}=1 \mathrm{~g} / \mathrm{mL}\right)$ podemos decir que:

$$
\text { masa total de disolvente }=30 \mathrm{~g}+\text { masa de disolvente agregada. }
$$

O sea, que para calcular la cantidad de agua agregada habría que restar los $30 \mathrm{~g}$ que inicialmente se utilizaron para preparar la disolución $\mathrm{N}^{\circ} 4$ :

$$
271,83 \mathrm{~g}-30 \mathrm{~g}=241,83 \mathrm{~g} .
$$

Teniendo en cuenta que el disolvente a agregar es agua y que su densidad es de $1 \mathrm{~g} / \mathrm{mL}$, el volumen de agua que debería agregar a la disolución $\mathrm{N}^{\circ} 4$ para que la concentración de la nueva dilución fuera igual a la de la disolución $\mathrm{N}^{\circ} 1$ sería de 241,83 $\mathrm{mL}$.

Se comprobó experimentalmente el cálculo realizado, agregando dicho volumen de agua a la disolución $\mathrm{N}^{\circ} 4$ y comparando la coloración de la dilución obtenida con la de la disolución $\mathrm{N}^{\circ}$ 1 preparada inicialmente.

En esta primera parte experimental se pudieron trabajar los conceptos asociados al tema disoluciones como: soluto y disolvente, disoluciones diluidas y concentradas, diluciones, densidad, composición y concentración de una disolución.

Segunda parte: Análisis cualitativo: la cromatografía como método de fraccionamiento de los componentes de una disolución.

De manera general, se puede definir la cromatografía como una técnica que permite la separación de los componentes de una mezcla debido a la influencia de dos efectos contrarios: la retención y el desplazamiento. La retención es el efecto ejercido sobre los componentes de la mezcla por una fase estacionaria, que puede ser un sólido o un líquido anclado a un soporte sólido; mientras que el desplazamiento es el efecto ejercido sobre los componentes de la mezcla por una fase móvil, que puede ser un líquido o un gas (García Csákÿ y Martínez Grau 2012).

El análisis cualitativo de los colorantes presentes en el jugo en polvo sabor naranja en estudio, se realizó mediante cromatografía en papel. La separación de los colorantes se evidenció por las diferentes velocidades con que son arrastrados cada uno de ellos a través de un medio poroso (fase estacionaria) por un disolvente en movimiento (fase móvil) que asciende por capilaridad. Como no todos son arrastrados con la misma velocidad, al cabo de un rato se forman franjas de colores que corresponden a los colorantes a identificar.

Para el desarrollo de la cromatografía se utilizó papel de filtro como fase estacionaria. En particular se usó Papel Cualitativo Filtrado Medio (N 102) de 110 mm de diámetro. 
Con respecto a la fase móvil, se analizaron dos eluyentes (agua y etanol) para seleccionar aquel que permitiera una adecuada separación de los componentes de la mezcla. Se escogió etanol como el eluyente más apropiado para realizar la cromatografía debido a que, al utilizar agua, se observó mayor turbidez en la disolución preparada y, para evitar la proliferación de microorganismos. Asimismo, se consideró que el eluyente empleado cumpliera con los principios de la Química Verde.

La Química Verde, también denominada Química Sustentable, es la química para la prevención de la contaminación. Se ha definido como el diseño, la manufactura y el uso de sustancias químicas y procesos que reducen o eliminan el uso o la generación de residuos y productos nocivos para el medio ambiente o la salud humana. Los 12 principios de la Química Verde o Sustentable fueron formulados por Paul Anastas y John Warner (1998, p. 30).

La muestra se preparó en un vaso de precipitados de $100 \mathrm{~mL}$ disolviendo 1 gramo de jugo de naranja en polvo en $10 \mathrm{~mL}$ de etanol $96^{\circ}$. Luego se colocó en su interior una tira de papel de filtro de $2 \mathrm{~cm} \times 5 \mathrm{~cm}$ y se dejó correr el eluyente hasta verificar la separación de los componentes de la muestra. A los fines prácticos, para un análisis cualitativo de los colorantes, este procedimiento resultó el más conveniente ya que permitió distinguir a simple vista los componentes coloreados presentes en el jugo, sin necesidad de emplear el método de sembrado. Dicho método consiste en colocar la muestra disuelta, utilizando un capilar de vidrio o una pipeta Pasteur, en un punto de aplicación marcado sobre una línea trazada a aproximadamente $1 \mathrm{~cm}$ del borde inferior de la placa. Si la muestra se encuentra muy diluida, deberá repetirse el proceso.

Los colorantes presentes en la marca de jugo en polvo escogida son dióxido de titanio, tartrazina y amarillo ocaso (tabla 2). La experiencia permitió separar e identificar dos de los tres colorantes encontrados en el jugo en polvo de la marca escogida ya que el dióxido de titanio es un polvo blanco, insoluble en agua y en etanol y por lo tanto no pudo determinarse por este método.

Tabla 2. Color y solubilidad de los colorantes presentes en el sobre de jugo en polvo sabor a naranja elegido para su estudio.

\begin{tabular}{|c|c|}
\hline Colorante & Color del colorante \\
\hline Dióxido de titanio & Blanco \\
\hline Tartrazina & Amarillo intenso \\
\hline Amarillo Ocaso & Naranja claro \\
\hline
\end{tabular}

Para la posterior identificación y determinación de los colorantes presentes en la placa cromatográfica, se repartieron a los diversos grupos dos corridas cromatográficas realizadas con amarillo ocaso y tartrazina puros, las cuales fueron utilizadas como patrones de referencia.

En la figura 1 se muestran los patrones de referencia (A) y (B) correspondientes a los colorantes amarillo ocaso y tartrazina respectivamente junto con la placa cromatográfica de una muestra de jugo en polvo sabor naranja, obtenida por uno de los grupos (C).

La cromatografía también se utiliza como técnica analítica para identificar los componentes de una muestra, mediante la comparación de sus valores de $R_{\mathrm{f}}$ (del inglés retention factor). El $R_{\mathrm{f}}$ es constante para cada sustancia y es una expresión del movimiento relativo de la sustancia respecto del disolvente. Puede calcularse utilizando la fórmula $R_{\mathrm{f}}=\mathrm{dm} / \mathrm{ds}$, donde $\mathrm{dm}=$ distancia recorrida por la muestra y ds $=$ distancia recorrida por el disolvente.

Para los objetivos propuestos, meramente cualitativos, no resultó necesario sembrar la muestra con capilar ni realizar cálculos de $R_{\mathrm{f}}$ 


\section{Colorantes alimentarios y su relación con la salud.}

El objetivo de esta propuesta es el de reflexionar acerca de los posibles efectos adversos de los colorantes alimentarios para la salud del consumidor y el nivel de toxicidad asociado a los mismos. Para realizar esta actividad se dispone la clase en grupos a cada uno de los cuales se les entregan tres hojas con información de los colorantes presentes en el jugo de naranja en polvo en estudio, a saber: dióxido de titanio, tartrazina y amarillo ocaso. La información, la cual detalla la descripción del aditivo, usos, otros nombres, efectos secundarios y nivel de toxicidad fue tomada de la página web Aditivos alimentarios (s.f.)

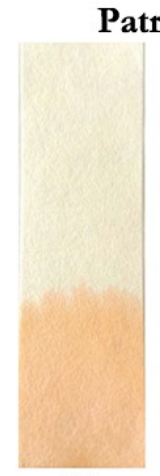

(A)
Patrones

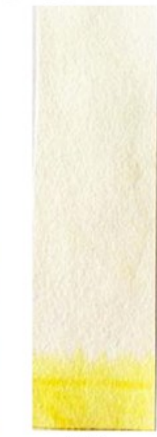

(B)

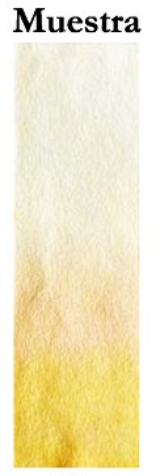

(C)

Figura 1. Fotografías de las cromatografías en papel de los patrones amarillo ocaso (A) y tartrazina (B), y de la muestra de jugo en polvo sabor naranja (C).

Se les propone a los/las estudiantes que, con la ayuda del material disponible y buscando en internet aquella información que crean necesaria, realicen las siguientes actividades de investigación.

\section{Primera consigna:}

Respecto a los colorantes en estudio investiga: qué otros nombres reciben, cuál es su fórmula química, cuál es su estructura química y qué tipo de compuestos son.

Responde la consigna realizando una tabla que permita comparar los datos investigados.

La tabla 3 presenta la información recabada por los/las estudiantes respecto a los colorantes en estudio presentes en un jugo de naranja en polvo, de acuerdo a los requerimientos de la primera consigna (otros nombres, fórmula química, estructura química y tipo de compuesto).

La investigación fue puesta en común y consensuada por los diferentes grupos.

Desde el punto de vista químico, se hizo especial hincapié en el análisis de las estructuras químicas de los tres colorantes alimentarios en estudio estableciendo similitudes y diferencias que permitieran inferir acerca de sus comportamientos. 
Tabla 3. Información recabada por los/las estudiantes respecto a los colorantes en estudio presentes en un jugo de naranja en polvo, de acuerdo a los requerimientos de la primera consigna (otros nombres, fórmula química, estructura química y tipo de compuesto).

\begin{tabular}{|c|c|c|}
\hline Colorante & Otros nombres & $\begin{array}{l}\text { Fórmula química, estructura química } \\
\text { y tipo de compuesto }\end{array}$ \\
\hline Tartrazina & $\begin{array}{l}\text { E102 (Unión Europea) } \\
\text { INS } 102 \text { (Sistema Internacional de } \\
\text { Numeración, Codex Alimentarius } \\
\text { FAO/OMS) } \\
\text { Amarillo 5, Amarillo Ácido 23, Amarillo } \\
\text { Alimentario } 4 \text { (en la mayoría de los países } \\
\text { hispanoparlantes) } \\
\text { Yellow 5, Acid Yellow 23, Food yellow } 4 \\
\text { (FDA-USA) } \\
\text { C.I. } 19140 \text { (Colour Index International) }\end{array}$ & $\begin{array}{l}\text { Fórmula química: } \mathrm{C}_{16} \mathrm{H}_{9} \mathrm{~N}_{4} \mathrm{Na}_{3} \mathrm{O}_{9} \mathrm{~S}_{2} \\
\text { Estructura química: } \\
\text { Tipo de compuesto: Compuesto orgánico. } \\
\text { Colorante azoico } \\
\quad \text { (contienen el grupo azo -N=N-) }\end{array}$ \\
\hline $\begin{array}{c}\text { Amarillo } \\
\text { ocaso }\end{array}$ & $\begin{array}{l}\text { E110 (Unión Europea) } \\
\text { INS } 110 \text { (Sistema Internacional de } \\
\text { Numeración, Codex Alimentarius FAO/ } \\
\text { OMS) } \\
\text { amarillo crepúsculo, amarillo ocaso FCF, } \\
\text { amarillo No } 6 \text { (en la mayoría de los países } \\
\text { hispanoparlantes) } \\
\text { Sunset Yellow FCF, Orange Yellow S, } \\
\text { Yellow } 6 \text { (FDA-USA) } \\
\text { C.I. } 15985 \text { (Colour Index International) }\end{array}$ & $\begin{array}{l}\text { Fórmula química: } \mathrm{C}_{16} \mathrm{H}_{10} \mathrm{~N}_{2} \mathrm{Na}_{2} \mathrm{O}_{7} \mathrm{~S}_{2} \\
\text { Estructura química: } \\
\text { Tipo de compuesto: Compuesto orgánico. } \\
\text { Colorante azoico } \\
\text { (contienen el grupo azo }-\mathrm{N}=\mathrm{N}-\text { ) }\end{array}$ \\
\hline $\begin{array}{l}\text { Dióxido de } \\
\text { titanio }\end{array}$ & $\begin{array}{l}\text { Nomenclatura sistemática: dióxido de } \\
\text { titanio } \\
\text { Nomenclatura stock: óxido de titanio (IV) } \\
\text { Nomenclatura tradicional: óxido titánico }\end{array}$ & $\begin{array}{l}\text { Fórmula química: } \mathrm{TiO}_{2} \\
\text { Estructura química: } \mathrm{O}=\mathrm{Ti}=\mathrm{O} \\
\text { Tipo de compuesto: Compuesto inorgánico } \\
\quad \text { Óxido metálico (metal + oxígeno) }\end{array}$ \\
\hline
\end{tabular}

\section{Segunda consigna:}

Se propuso a los/las estudiantes realizar una tabla listando comparativamente, los efectos adversos para la salud documentados y la Ingesta Diaria Admisible (IDA) autorizada, para cada colorante en estudio, por el comité FAO/OMS (Organización de las Naciones Unidas para la Agricultura y la Alimentación/Organización Mundial de la Salud).

La ingesta diaria admisible (IDA) es la cantidad aproximada de aditivo presente en un determinado alimento, expresada en miligramos de aditivo en relación con la masa corporal $\mathrm{del} /$ la consumidor/a y que se puede ingerir a diario, durante toda la vida de una persona, sin que llegue a representar un riesgo significativo para la salud (Comisión del Codex Alimentarius 2012).

Este índice que informa acerca del grado de peligrosidad de la ingesta de un aditivo alimentario protege la salud de los consumidores y es regulado por comités científicos de expertos nacionales e internacionales.

La tabla 4 detalla la Ingesta Diaria Admisible y los efectos secundarios que pueden originar los colorantes en estudio, ya sea por su consumo excesivo o por la hipersensibilidad natural de quien lo consuma.

De acuerdo a lo estipulado en el Código Alimentario, los colorantes tartrazina y amarillo ocaso en bebidas no deben exceder la siguiente concentración: 0,010 g colorante/100 g bebida.

Con respecto al colorante dióxido de titanio en bebidas se indica quantum satis. Este término latino significa la cantidad adecuada. 
Tabla 4. Efectos secundarios, advertencias e Ingesta Diaria Admisible (IDA) de los colorantes tartrazina, amarillo ocaso y dióxido de titanio.

\begin{tabular}{|c|c|c|}
\hline Colorante & Efectos secundarios/ Advertencias & Ingesta Diaria Admisible (IDA) \\
\hline Dióxido de titanio & $\begin{array}{l}\text { En dosis bajas puede teñir las uñas de color } \\
\text { amarillo. En grandes dosis puede bloquear la } \\
\text { respiración celular, en órganos como el hígado } \\
\text { y riñones. } \\
\text { En test realizados con animales, se demostró } \\
\text { que sus nanopartículas provocan daños } \\
\text { cromosómicos y aumentan la probabilidad de } \\
\text { sufrir riesgo de cáncer. }\end{array}$ & No especificado $(*)$ \\
\hline Tartrazina & $\begin{array}{l}\text { Puede ocasionar hiperactividad en niños, } \\
\text { aumentar los síntomas del asma, producir } \\
\text { eczemas, urticaria y provocar insomnio. } \\
\text { Al ser un colorante azoico, puede provocar } \\
\text { reacciones en personas alérgicas al ácido } \\
\text { salicílico. } \\
\text { Su consumo a largo plazo podría ser } \\
\text { cancerígeno. }\end{array}$ & $7,5 \mathrm{mg} / \mathrm{kg}$ de masa corporal \\
\hline Amarillo ocaso & $\begin{array}{l}\text { Puede ocasionar hiperactividad en niños, } \\
\text { aumentar los síntomas del asma, producir } \\
\text { eczemas, urticaria y provocar insomnio. } \\
\text { Al ser un colorante azoico, puede provocar } \\
\text { reacciones en personas alérgicas al ácido } \\
\text { salicílico. } \\
\text { Su consumo a largo plazo podría ser } \\
\text { cancerígeno. }\end{array}$ & $2,5 \mathrm{mg} / \mathrm{kg}$ de masa corporal \\
\hline
\end{tabular}

* "Debe tenerse presente que una IDA no especificada no significa que se acepta una ingestión ilimitada" (Codex Alimentarius 1995).

Luego de discutir respecto al IDA y a la cantidad adecuada de cada alimento en el envase de jugo en polvo se les proponen a los/las estudiantes los siguientes tres desafíos.

\section{Primer desafio:}

Un sobre de jugo en polvo para preparar 1 litro de bebida contiene 18 gramos. Teniendo en cuenta que rinde 4 vasos de jugo, ¿cuántos miligramos de tartrazina habría en un vaso de jugo si se considera que la concentración máxima de tartrazina en dicha bebida es de 0,010 g tartrazina/ $100 \mathrm{~g}$ jugo en polvo?

$18 \mathrm{~g}$ jugo en polvo $\times 0.010 \mathrm{~g}$ tartrazina $/ 100 \mathrm{~g}$ jugo en polvo $=0,0018 \mathrm{~g}$ tartrazina

$$
0,0018 \mathrm{~g} \text { tartrazina } \times 1000 \mathrm{mg} / 1 \mathrm{~g}=1,8 \mathrm{mg} \text { tartrazina. }
$$

Los 1,8 mg de tartrazina estarán en 1 litro de bebida preparada, si consideramos que por cada litro obtenemos 4 vasos:

$$
1,8 \mathrm{mg} / 4 \text { vasos }=0,45 \mathrm{mg} \text { tartrazina/ vaso. }
$$

Es decir que en cada vaso habrá como máximo $0,45 \mathrm{mg}$ de tartrazina disueltos.

Análogamente podríamos hacer el mismo cálculo para el amarillo ocaso en cuyo caso el resultado sería el mismo dado que la concentración máxima permitida en bebidas es igual a la de la tartrazina $(0,010 \mathrm{~g}$ colorante $/ 100 \mathrm{~g}$ bebida $)$.

En el caso del dióxido de titanio no se puede hacer este cálculo por estar contenido quantum satis. 


\section{Segundo desafio:}

Teniendo en cuenta los valores de IDA encontrados para los colorantes tartrazina y amarillo ocaso (tabla 4) y tu masa corporal, ¿cuánto podrías consumir a diario de cada colorante, durante toda tu vida, sin que llegue a representar un riesgo significativo para tu salud?

De la tabla 4 vemos que:

IDA tartrazina $=7,5 \mathrm{mg} / \mathrm{kg}$ de masa corporal,

IDA amarillo ocaso $=2,5 \mathrm{mg} / \mathrm{kg}$ de masa corporal.

Suponiendo una masa corporal de $60 \mathrm{~kg}$, el cálculo para el colorante tartrazina será:

$60 \mathrm{~kg}$ de masa corporal $\times 7,5 \mathrm{mg}$ tartrazina $/ \mathrm{kg}$ de masa corporal $=450 \mathrm{mg}$ tartrazina

Podría decirse que: "si mi masa corporal fuera de $60 \mathrm{~kg}$, podría consumir $450 \mathrm{mg}$ de amarillo ocaso por día durante toda la vida, sin que llegue a representar un riesgo significativo para la salud".

Análogamente, para el amarillo ocaso:

$60 \mathrm{~kg}$ de masa corporal $\times 2,5 \mathrm{mg}$ amarillo ocaso $/ \mathrm{kg}$ de masa corporal $=150 \mathrm{mg}$ amar. ocaso.

Es decir, una persona cuya masa corporal fuera de $60 \mathrm{~kg}$, podría consumir $150 \mathrm{mg}$ de amarillo ocaso por día durante toda la vida, sin que llegue a representar un riesgo significativo para la salud.

Tercer desafio:

La tabla 5 muestra los valores de masa corporal promedio y talla para niños y niñas de entre 5 y 8 años de edad establecidos por la OMS.

Tabla 5. Masas corporales promedio y talla establecida por la OMS para niños y niñas de entre 5 y 8 años de edad.

\begin{tabular}{|c|c|}
\hline Edad & Masa corporal promedio \\
\hline 5 años & $18,03 \mathrm{~kg}$ \\
\hline 6 años & $19,91 \mathrm{~kg}$ \\
\hline 7 años & $22 \mathrm{~kg}$ \\
\hline 8 años & $23,56 \mathrm{~kg}$ \\
\hline
\end{tabular}

Teniendo en cuenta los valores de IDA encontrados para los colorantes tartrazina y amarillo ocaso (tabla 4) y la masa corporal de estos niños y niñas, ¿cuánto podrian consumir a diario de cada colorante, durante todas sus vidas, sin que llegue a representar un riesgo significativo para su salud? Compara los resultados con los obtenidos teniendo en cuenta tu masa corporal.

Como ejemplo se realizan los cálculos teniendo en cuenta la masa corporal de un/a niño/a de 5 años.

Para la tartrazina:

$18,03 \mathrm{~kg}$ de masa corporal $\times 7,5 \mathrm{mg}$ tartrazina $/ \mathrm{kg}$ de masa corporal $=135,225 \mathrm{mg}$.

Para el amarillo ocaso:

$18,03 \mathrm{~kg}$ de masa corporal $\times 2,5 \mathrm{mg}$ amarillo ocaso $/ \mathrm{kg}$ de masa corporal $=45,075 \mathrm{mg}$. Análogamente se realiza el cálculo para el resto de los niños/as. 
La tabla 6 muestra de manera comparativa los valores obtenidos para cada colorante alimenticio presente en jugo en polvo sabor naranja, en función de las masas corporales de los consumidores.

Tabla 6. Tabla comparativa de los resultados obtenidos en el tercer desafí.

\begin{tabular}{|c|c|c|}
\hline Edad & Masa corporal promedio & Consumo diario de tartrazina por día durante toda la vida* \\
\hline 5 años & $18,03 \mathrm{~kg}$ & $135,225 \mathrm{mg}$ \\
\hline 6 años & $19,91 \mathrm{~kg}$ & $149,325 \mathrm{mg}$ \\
\hline 7 años & $22 \mathrm{~kg}$ & $165 \mathrm{mg}$ \\
\hline 8 años & $23,56 \mathrm{~kg}$ & $176,7 \mathrm{mg}$ \\
\hline 13 años & $60 \mathrm{~kg}$ & $450 \mathrm{mg}$ \\
\hline
\end{tabular}

* Sin que llegue a representar un riesgo significativo para su salud

\section{Sondeo y sociabilización de lo trabajado}

Con el fin de socializar lo discutido y aprendido en las actividades teórico-prácticas y generar concientización, los/las estudiantes elaboraron una encuesta acerca de los colorantes alimentarios. Los objetivos de la misma fueron: conocer el nivel de información que presenta la comunidad educativa con respecto a este tipo de sustancias, el conocimiento o desconocimiento de los efectos adversos en la salud, la frecuencia en la lectura del etiquetado por parte de los consumidores, el preparado de disoluciones alimenticias, entre otros. La encuesta fue realizada en formularios de Google Drive y difundida mediante un link al que se pudo acceder fácilmente desde celulares, computadoras, etcétera. Posteriormente, se realizó el análisis estadístico de los resultados, que se plasmaron en tablas y gráficos. Se empleó dicha encuesta como una instancia de reflexión de los contenidos trabajados durante el desarrollo de la propuesta. Finalmente, a partir de la información recabada en las encuestas, se trabajó de manera grupal en la realización de afiches informativos con el fin de generar conciencia en el resto de la comunidad educativa acerca de la presencia de colorantes en las disoluciones preparadas y consumidas a diario.

\section{Resultados y conclusiones}

A través del trabajo contextualizado y de las actividades teórico-prácticas propuestas, se logró el abordaje del tema disoluciones poniendo en consideración el estudio de los colorantes alimentarios presentes en jugos en polvo y su relación con la salud.

La estrategia metodológica aplicada resultó útil para sortear las dificultades que los/las estudiantes suelen manifestar al abordar el tema en estudio y para enfatizar la importancia del trabajo experimental y la contextualización.

Además de trabajar con los conceptos asociados al tema disoluciones como: soluto y disolvente, disoluciones diluidas y concentradas, diluciones, densidad, composición y concentración de una disolución, se logró identificar a los colorantes presentes en el jugo en polvo en estudio utilizando la cromatografía como método de fraccionamiento de los componentes de una disolución.

Las estrategias implementadas hicieron posible la participación activa de los/las estudiantes en las diferentes propuestas teórico-prácticas, quienes demostraron interés y apertura hacia el trabajo grupal. Se trabajó de manera cooperativa respetando los aportes tanto individuales como colectivos. 
Los desafíos planteados y las reflexiones realizadas permitieron a los/las estudiantes comprobar que todos los colorantes presentes en el jugo en polvo en estudio presentan un cierto nivel de toxicidad que debe ser tenido en cuenta y que están asociados a diversos efectos adversos para la salud, principalmente en niños.

Consideramos imprescindible el abordaje de la educación para la salud y para el consumidor como un contenido transversal en las clases de ciencias. Resulta fundamental, fomentar el hábito de lectura de las etiquetas de los alimentos consumidos ya que sólo en la medida en que los/las adolescentes sean capaces de reconocer y comprender la información que brindan los rótulos como así también de identificar las sustancias potencialmente nocivas para la salud, podrán constituirse en consumidores responsables y, a su vez, transmitir la información aprendida a sus familiares y pares; constituyéndose en agentes multiplicadores del saber construido.

A través de las producciones de los/las estudiantes, de las conclusiones plasmadas y de las entrevistas realizadas se pudo evidenciar un aprendizaje consistente con los objetivos planteados.

\section{Agradecimientos}

Las autoras agradecen a la Universidad Nacional del Sur el financiamiento del proyecto de investigación acreditado "Estudios de química en contexto desde un enfoque Ciencia Tecnología y Sociedad (CTS)", dirigido por la Dra. Sandra A. Hernández, en el marco del cual se realizó este trabajo.

\section{Referencias}

Aditivos alimentarios (s.f.) Consultado en línea en https:/ /www.aditivos-alimentarios.com/

Anastas P., Warner J. (1998) Green Chemistry: Theory and Practice. New York: Oxford University Press.

Ausubel D. (1963) The psychology of meaningful verbal learning. New York: Grune and Stratton.

Blanco López A., Prieto Ruz T. (1994) Las disoluciones: dificultades de aprendizaje y sugerencias para su enseñanza. Alambique. Didáctica de las Ciencias Experimentales 1, 125131.

Brizuela L., Hernández S. (2016) Bebidas alternativas al consumo de agua de red. The Journal of the Argentine Chemical Society 103 (1-2), 1-3.

Broman K., Parchmann I. (2014) Students' application of chemical concepts when solving chemistry problems in different contexts. Chemistry Education Research and Practice 15 (4), 516-529.

Buera M. (2011) La química, el color, los alimentos y el arte. Capítulo 27 (pp. 263-274) en Química y Civilización. Lydia Galagovsky (directora). Ciudad Autónoma de Buenos Aires: Asociación Química Argentina.

Burrows J. (2009) Palette of Our Palates: A Brief History of Food Coloring and Its Regulation. Comprehensive Reviews in Food Science and Food Safety 4, 394-408.

Caamaño A. (2018) Enseñar química en contexto: un recorrido por los proyectos de química en contexto desde la década de los 80 hasta la actualidad. Educación quimica 29 (1), 21-54.

Cheeseman M. A. (2012) Artificial Food Color Additives and Child Behavior. Environmental Health Perspectives 120 (1), a15-a16.

Codex Alimentarius (1995) Norma general para los aditivos alimentarios. Codex Stan 192-1995.

Codex Alimentarius (2007) Etiquetado de los alimentos, $5^{\text {a }}$ ed. FAO/OMS: Roma. 
Comisión del Codex Alimentarius (2012) Programa conjunto de la FAO/OMS sobre normas alimentarias comité del Codex sobre aditivos. CX/FA 12/44/2. 44ª reunión Hangzhou, China, 12 al 16 de marzo de 201.

Cúneo F., Schaab N. (2013) Hábitos de consumo de bebidas en adolescentes y su impacto en la dieta. Diaeta 31 (142), 34-41.

Díaz-Barriga F., Hernández Rojas G. (2002) Estrategias docentes para un aprendiraje significativo. Una interpretación constructivista. México: McGraw-Hill.

García Csaky A., Martinez Grau M. (2012). Técnicas experimentales en sintesis orgánica, $2^{\mathrm{a}}$ ed. Madrid: Editorial Síntesis.

Johnstone A. H. (1991) Why is science difficult to learn? Things are seldom what they seem. Journal of Computer Assisted Learning 7, 75-83.

León Espinosa de los Monteros M. T., Rueda Domingo M. T., Castillo Sánchez M. D., León Espinosa de los Monteros M., Ceballos Atienza R., Fernández Lloret S. (2000) Estudio de los aditivos alimentarios y su repercusión en la población infantil. Medicina de Familia. Andalucía 1, 25-30

Limón M., Carretero M. (1997) Las ideas previas de los alumnos: ¿qué aporta este enfoque a la enseñanza de las Ciencias? pp. 3-18 en M. Carretero (comp.), Construir y enseñar: las Ciencias Experimentales. Buenos Aires: Aique.

Nappa N., Insausti M. J., Sigüenza A. F. (2005) Obstáculos para generar representaciones mentales adecuadas sobre la disolución. Revista Eureka sobre Enseñanza y Divulgación de las Ciencias 2 (3), 344-363.

Marchán-Carvajal I., Sanmartí N. (2014) Una revisión sobre el uso de contextos en la enseñanza de las ciencias y su potencial para el desarrollo de la competencia científica. pp. 702-710 en De las Heras et. al. (coord.), Investigación y transferencia para una educación en ciencias: Un reto emocionante. Huelva: Servicio de Publicaciones de la UHU.

OMS/FAO (2007) Etiquetado de los Alimentos (Codex Alimentarius), $5^{\text {a }}$ ed. Roma: FAO.

Ricchi G., Torres N. (2014) Disoluciones: ¿Contribuye la experimentación a un aprendizaje significativo? Educación Quimica 25 (1), 21-29.

Ruiz Hidalgo J. (2009) Didáctica del contenido transversal educación para el/la consumidor/a en las materias de física y química en la ESO y el bachillerato. Revista Digital Innovación y experiencias educativas $\mathrm{N}^{\circ} 20$ (julio).

Sánchez Juan R. (2013) La química del color en los alimentos. Química Viva 12 (3), 234-246.

Sanmartí Puig N., Márquez Bargalló C. (2017) Aprendizaje de las ciencias basado en proyectos: del contexto a la acción. Apice. Revista de Educación Científica 1 (1), 3-16. 